\title{
Guido Gozzano: Dal Decadentismo al cammino moderno della Poesia Italiana
}

\author{
Giorgia MARANGON \\ Departamento de Traducción e Interpretación, \\ Lenguas Romances, Estudios Semíticos y Documentación \\ Universidad de Córdoba \\ lr1marmg@uco.es
}

\begin{abstract}
RiaSSUNTO
In questo articolo si definisce e analizza l'excursus letterario e poetico che si sviluppa dal Decandentismo fino al cammino moderno della poesia italiana, risaltando la figura artistica di Guido Gozzano. Tra la fine dell'Ottocento e gli inizi del Novecento i nomi di Giovanni Pascoli e Gabriele D'Annunzio fanno grande la poesia italiana. La svolta arriva con i crepuscolari; l'Italia si affaccia all'epoca moderna attraverso la poetica, il linguaggio e le tematiche di Guido Gozzano.
\end{abstract}

Parole chiave: Decadentismo, età moderna, crepuscolari, Gozzano, poetica degli oggetti.

[Recibido, diciembre 2010; aprobado, marzo 2011]

Guido Gozzano: from Decadentism to the modernity of Italian poetry

\begin{abstract}
This article defines and analyzes the literary and poetic excursus that passes since the decadentism to the modernity of the italian poetry, emphasizing the artistic figure of Guido Gozzano. Between the final of the eight hundred and the principle of the nine hundred the names of Giovanni Pascoli and Gabriele D'Annuzio become the Italian poetry exalted. The crepuscolari make the change; Italy opens to the modernity across the poetry, the language, and the thematic of Guido Gozzano.
\end{abstract}

Keywords: Decadentism, modernity, crepuscolari, Gozzano, objects’ poerty. 


\section{Introduzione: La poetica dei crepuscolari}

Accadeva in fondo quello che accade ad ogni generazione, in cui alcune forme di poesia predominano e vengono ripetute dai più, un poco dietro l'influsso di chi per loro mezzo ha saputo raggiungere l'arte o più semplicemente la fama, un poco perché una comune disposizione spirituale fa sentire quelle forme come le più adatte all'estrinsecazione del proprio stato d'animo: si formano così quelle che noi chiamiamo scuole o movimenti letterari, ma che in realtà solo difficilmente e solo fino ad un certo punto sono letterari, ché per lo più sono invece l'eco e il riflesso di movimenti spirituali, i quali determinano non solo la scelta dei temi, ma persino ciò che si suole chiamare la tecnica. (Petronio 1937: 5-6)

Nella storia del Decadentismo italiano si iscrivono due movimenti poetici che diversamente riprendono e superano le esperienze rappresentate dalla poesia pascoliana e dannunziana. Si tratta del gruppo dei crepuscolari e di quello dei futuristi, il primo più riservato e alieno da proclamazioni clamorose della propria novità, il secondo più aggressivo e polemico nella sua risoluta lotta contro il passato e nella propaganda combattiva e fragorosa delle sue vistose innovazioni tecniche e della sua visione moderna della poesia e della vita.

Il termine "crepuscolare" venne usato per la prima volta da un critico, Giuseppe Antonio Borgese ${ }^{1}$, in quanto i crepuscolari appunto gli sembravano rappresentare il crepuscolo della grande giornata di poesia vissuta dalla letteratura italiana fino a quel momento. I crepuscolari elevano a materia della loro poesia la vita quotidiana nei suoi aspetti più umili e banali, togliendo però alle "piccole cose" quel valore simbolico che era proprio del Pascoli. I crepuscolari svuotano il simbolo della centralità che aveva in Pascoli facendolo diventare scenario di pessimo gusto della realtà che raccontano. Il confronto tra le due realtà è d'obbligo; da una parte il maestro, Pascoli, interprete delle cose, traduttore del linguaggio delle cose e della natura e, dall'altra la scelta dissacrante, consapevole e calcolata della diminuzione del tono, del messaggio, dello spazio, della parola. Il simbolismo che si fonda sulla tendenza a caricare di valore simbolico gli oggetti (vedi la poesia Lavandare: "l'aratro in mezzo alla maggese" (Budriesi, 1990, p. 59) che esprime tutta la solitudine, l'abbandono, la malinconia di chi è lontano dalla parsona che ama; la casa che simboleggia il nido, la culla, la madre, simbolo di protezione, di regressione all'infanzia, di continuità ciclica dell'esistenza: vita / morte) lascia spazio alle piccole cose di pessimo gusto, elemento chiave della poetica crepuscolare.

Così il ritmo della vita quotidiana si presenta in tutto il suo grigiore sonnolento, stanco, vuoto di ogni ideale superiore, privo di ogni slancio ed entusiasmo. L'aridità, la sfiducia, la noia, l'incapacità di dare un significato alla vita sono gli stati d'animo più soliti: è un'esistenza malinconica, talora fra

\footnotetext{
${ }^{1}$ Vedi articolo Poesia crepuscolare, in La Stampa, $1^{\circ}$ setiembre 1910.
} 
ospedali, suore, organetti, vecchie ville, malati; si respira lo spleen ${ }^{2}$ dei giorni di festa, il suono mesto delle campane che scandisce il trascorrere del tempo con i suoi rintocchi. Su tutto questo può innestarsi talvolta il sogno di qualcosa per cui valga la pena di vivere, accompagnato però dalla coscienza che si tratta di un sogno.

Al di qua dell'azione, e come tagliati fuori dai moti e dai conflitti reali della storia, i crepuscolari vivono la loro delusione esistenziale e storica di piccoli borghesi in negativo, in una condizione limbale di indecisione e di accidia, tra adesione e negazione della quotidianità. (Savoca 1985: 91)

Diviene così fondamentale l'ironia, come continua consapevolezza della vanità delle illusioni $\mathrm{e}$, insieme, della vacuità della vita. I crepuscolari spesso arrivano, soprattutto con Guido Gozzano, ad un equilibrio difficile tra sorriso ed affetto, tra rimpianto e piacere trasgressivo, fra ironia e abbandono alla banalità. C'è incertezza fra passato e futuro, fra chiusura e apertura. A tali contenuti corrisponde anche una consapevole rivoluzione formale. I crepuscolari, infatti, tendono alla riduzione della poesia a prosa, il loro verso tende ad una poesia che si mantenga nell'ambito della prosa. La lingua aulica della tradizione poetica dannunziana si abbassa fino a includere il lessico della banalità, della quotidianità, l'understatement $\mathrm{o}$ abbassamento di tono verso l'usuale.

Quel complesso di stati d'animo e di atteggiamenti mentali che si è convenuto di definire condizione crepuscolare giunge alla più autentica cifra artistica nell'opera di Guido Gustavo Gozzano (1883-1916).

\section{Guido Gozzano tra cultura e decadenza}

Dopo una così esasperata tensione verso un'esistenza superumana, dopo una ricerca di vite eccezionali e inimitabili, quale è quella che il D'Annunzio cantava e voleva vivere (si ricordino le parole del protagonista de Il Piacere, Andrea Sperelli: "bisogna fare la propria vita come si fa un'opera d'arte" (Budriesi 1990: 157), la poesia di Gozzano appare un richiamo alla realtà quotidiana. Nella sua poesia si afferma per la prima volta l'atteggiamento del poeta disilluso di ogni cosa, sentimentalmente inaridito, ironicamente perplesso di fronte alla mutabilità dei fenomeni della vita, alla retorica, all'attivismo, all'egolatria del dannunzianesimo; sostanza del suo mondo poetico saranno argomenti provinciali e infantili, signorine un po' brutte, cose un po' vecchie, crinoline, ricami, colore rosa; ambiguità dell'amore senza passione, del sentimentalismo senza sentimento e dei profumi senza odori.

Il poeta è malato, ma la sua non è solo una malattia fisica: la vera malattia, dice continuamente Gozzano, è la tabes ${ }^{3}$ letteraria, l'irredimibile falsità della scrittura, l'impossibilità di far corrispondere la vita vera alla letteratura (a meno

\footnotetext{
${ }^{2}$ Vedi Les Fleurs du Mal di Charles Baudelaire.

${ }^{3}$ Tabes, is: consunzione, deperimento generato da malattie croniche.
} 
di non cadere nel ridicolo o nell'equivoco dell'estetismo). Tutta l'opera di Gozzano si forma su questa lucidissima coscienza del distacco fra letteratura e vita, coscienza amara e funebre che si trasforma in rictus ${ }^{4}$ umoristico, in quell'ironia che costituisce il tono primario dei suoi testi. Ed è proprio qui che scatta la distanza e il rifiuto del mito dannunziano: continuare a scrivere, per Gozzano, significa continuare a scrivere nella menzogna della letteratura, continuare a sorriderne, fare poesia sopra l'aridità della poesia, sull'impossibilità di avere una fede. La letteratura è il vuoto, una giocosa aridità larvata di chimere, imparentata non con la vita ma con la morte.

\subsection{Gozzano: il poeta delle "piccole cose"}

Gozzano riempie la sua poesia di oggetti-merce del presente e di oggettiricordo del passato, sottoponendoli alla corrosione ironica o alla sublimazione nostalgica. Gli oggetti che il poeta predilige sono costituiti da cose un tempo vive e poi uscite dal flusso della temporalità, per questo rimaste intatte, come appunto i quadri, il vasellame, i materassi e altro vario antiquariato della celebre soffitta del poema La signorina Felicita ovvero la Felicità (Gozzano 2008: 139158). L'amore per la ragazza di provincia è un luogo classico della narrativa ottocentesca; quanto al nome, non possiamo non ricordare che Félicité è la protagonista di Un coeur simple di Flaubert ${ }^{5}$.

IV.

Il nostro passo diffondeva l'eco

tra quei rottami del passato vano,

e la Marchesa dal profilo greco,

altocinta, l'un piede ignudo in mano,

si riposava all'ombra d'uno speco

arcade, sotto un bel cielo pagano.

Intorno a quella che rideva illusa

nel ricco peplo, e che morì di fame,

v'era una stirpe logora e confusa:

topaie, materassi, vasellame,

lucerne, ceste, mobili: ciarpame

reietto, così caro alla mia Musa!

Tra i materassi logori e le ceste

v'erano stampe di persone egregie;

incoronato dalle frondi regie

v'era Torquato nei giardini d'Este.

«Avvocato, perché su quelle teste

buffe si vede un ramo di ciliege?»

\footnotetext{
${ }_{5}^{4}$ Rictus-us: apertura della bocca, bocca aperta, spalancata (risata).

${ }^{5}$ Innumerevoli sono i riferimenti all'opera del francese. La stanza di Félicité è piena delle cose di pessimo gusto presenti nei versi di Gozzano.
} 
Io risi, tanto che fermammo il passo, e ridendo pensai questo pensiero:

Oimè! La Gloria! un corridoio basso, tre ceste, un canterano dell'Impero, la brutta effigie incorniciata in nero e sotto il nome di Torquato Tasso! (vv. 133-168)

Ne L'amica di nonna Speranza (Gozzano 2008: 159-167) c'è la descrizione nostalgico-ironica di un salotto del passato e di alcune figure risorgimentali e romantiche che vi si muovono: gli oggetti vi agiscono da consunti depositari dei valori del passato e da potenti evocatori di ricordi infantili, nostalgie e ironie storiche. Fiori e frutti in Gozzano sono così veri da sembrare finti e così finti da sembrare veri. I fiori non odorano perché sono finti e i frutti dai colori sgargianti sono di marmo, finti ma così belli da sembrare veri. Al primo verso troviamo il "Loreto impagliato", metonimia per uccello - al centro anche di alcune pagine di Un coeur simple di Flaubert - e il busto di Alfieri e Napoleone ${ }^{6}$. Da una parte Gozzano ricorda elencandoli tutti quegli oggetti che sono costitutivi dell'identità familiare e, dall'altra, li apostrofa come buone cose di pessimo gusto, quasi volesse estraniarsi da tutto ciò che lo circonda.

I.

Loreto impagliato ed il busto d'Alfieri, di Napoleone

i fiori in cornice (le buone cose di pessimo gusto),

il caminetto un po' tetro, le scatole senza confetti, i frutti di marmo protetti dalle campane di vetro,

un qualche raro balocco, gli scrigni fatti di valve, gli oggetti col monito, salve, ricordo, le noci di cocco,

Venezia ritratta a musaici, gli acquarelli un po' scialbi, le stampe, i cofani, gli albi dipinti d'anemoni arcaici,

le tele di Massimo d'Azeglio, le miniature, i dagherottìpi: figure sognanti in perplessità,

il gran lampadario vetusto che pende a mezzo il salone e immilla nel quarzo le buone cose di pessimo gusto,

il cùcu dell'ore che canta, le sedie parate a damasco chèrmisi... rinasco, rinasco del mille ottocento cinquanta! (vv. 1-14)

\footnotetext{
${ }^{6}$ Era tipico dell'Ottocento il gusto di tenere in casa i busti di personaggi storici e di artisti significativi.
} 


\subsection{Gozzano: ritratti femminili}

Il vero mondo di Gozzano si ritrova in quella ricca galleria di ritratti femminili che si apre con "bionda povera cosa" (v. 23) dal profumo disfatto / di mammole" (vv.17-18), "dall'occhio azzurro pervinca" (v. 24) di Un Rimorso (Gozzano, 2008, pp. 86-88). Oppure con la Graziella "ciclista" (v. 4) di Le due strade (Gozzano, 2008, pp. 102-107): "forte bella vivace bruna" (v. 22) e la "Signora" dalla "bocca vermiglia / troppo, le tinte ciglia" (vv. 43-44), "gli accesi dal veleno biondissimi capelli" (v. 47). Oppure Carlotta in L'amica di nonna Speranza: "lo sguardo al cielo profondo / e l'indice al labbro, secondo l'atteggiamento romantico" (vv. 105-106). Ciò che farà di Felicita la più popolare figura della poesia gozzaniana come Carlotta, come Graziella, è l'esattezza di un ambiente, di una stagione, di un mondo di oggetti e di costumi vagheggiati con elegante ironia e con affettosa delicatezza: ogni figura femminile di Gozzano respira questa stessa aura di minuzioso incanto, reca con sé questa atmosfera di evocazione intensa e puntuale. Carlotta rappresenta il desiderio di fuga dalla realtà della vita, rappresenta il passato - suscitato dal ritrovamento di una fotografia - "quel giorno - malinconica - vestivi un abito rosa / per farti, - novissima cosa! - ritrarre in fotografia" (vv. 107-108) che gli permetterebbe forse di poter "amare, amare d'amore" (v. 110), e ritrovare un se stesso diverso, capace di godere di quelle buone cose di pessimo gusto. Sono davvero di pessimo gusto quelle cose, e tutto quel mondo rimane pur sempre lontano e irreale, frivolo e artefatto, oggetto di uno sguardo disincantato e ironico che, mentre tenta l'abbandono, non può dimenticare le "margherite in collegio sfogliate" (v. 45) e la "romantica luna [...] che baci le chiome / dei pioppi, arcata siccome un sopracciglio di bimbo" (vv. 84-85). È una situazione paradossale coscientemente accettata: e al poeta non resta che fermare per il suo poetico conforto una serie mirabile di stampe, intensamente godute in questa loro specifica qualità. Amare d'amore non è possibile, oggi: non rimane che la coscienza del gioco fra l'intellettualistico e il dissacrante. Il presente è di ragazze come Ketty (Gozzano, 2008, pp. 241-245), "bel fiore del carbone e dell'acciaio" (v. 5), "fuma" (v. 6), "sputa" (v. 8), "cerulo - bionda, le mammelle assenti, ma forte come un giovinetto forte," (vv. 11-12), "signora di sé, della sua sorte" (v. 14), che gira il mondo prima di sposarsi con il cugino di Baltimora.

In Gozzano agisce la malinconia delle cose perdute e la dolcezza dei fantasmi del sogno. In Gozzano non resiste l'intensità viva delle personae se non calcolata sopra le voci del tempo e della lontananza. L'onirico incantesimo del tempo è appunto, nella poesia di Gozzano, l'anima vera delle sue personae come lo spirito vero dei suoi luoghi, e il trionfo del tempo è poi il solo trionfo che la sua poesia sia in grado di celebrare.

In La signorina Felicita ovvero la Felicità, storia forse autobiografica di un idillio giovanile ritrovato e vissuto con la memoria adulta rivolta alle cose, alle vicende che potevano essere e non sono state, Gozzano sostituisce all'amore sublime il vagheggiamento di una vita tranquilla insieme a una ragazza di provincia, casalinga e quasi brutta, ma rassicurante nella sua modesta 
semplicità. L'azzurro dei suoi occhi, che nella terminologia classica sono "iridi", non è più paragonato a quello del mare o del cielo ma il loro azzurro è "di stoviglia" (lessico della banalità). L'immagine femminile è quella tipica dell'universo poetico gozzaniano: una donna semplice, dedita alle attività domestiche, già abbozzata e parafrasata dal poeta in una lettera all'amica Amalia Guglielminetti del 3 agosto del 1907, in cui afferma di avere per compagna non una $\mathrm{Mila}^{7}$ ma una:

Servente indigena prosaicissima, [...] immaginate un corpo diciottenne, ma che in città sdegnerebbe una vecchia ottuagenaria, immaginate un volto quadrato, scialbo, roseo, lentigginoso, senza pupille, senza ciglia, senza sopracciglia, e un viscidume di capelli gialli, tirati, tirati lisci aderenti e stretti alla nuca in un fascio di trecciuole minute e su tutto il volto diffusi i segni dell'idiozia ereditaria. (Porecelli 1974: 122)

Anche Felicita è "quasi brutta" e nella descrizione che di lei fa Gozzano ritroviamo riflessa l'immagine della "servente" di cui sopra.

III.

Sei quasi brutta, priva di lusinga nelle tue vesti quasi campagnole, ma la tua faccia buona e casalinga, ma i bei capelli di color di sole, attorti in minutissime trecciuole, ti fanno un tipo di beltà fiamminga.

E rivedo la tua bocca vermiglia così larga nel ridere e nel bere, e il volto quadro, senza sopracciglia, tutto sparso d'efelidi leggiere e gli occhi fermi, l'iridi sincere azzurre d'un azzurro di stoviglia.

Tu m'hai amato. Nei begli occhi fermi rideva una blandizie femminina. Tu civettavi con sottili schermi, tu volevi piacermi, Signorina: e più d'ogni conquista cittadina mi lusingò quel tuo voler piacermi!

Ogni giorno salivo alla tua volta pel soleggiato ripido sentiero. Il farmacista non pensò davvero un'amicizia così bene accolta,

\footnotetext{
${ }^{7}$ La figlia di Iorio, celebre dramma di D'Annunzio (la meretrice Mila ne è la protagonista femminile).
} 
quando ti presentò la prima volta

l'ignoto villeggiante forestiero.

Talora - già la mensa era imbandita mi trattenevi a cena. Era una cena d'altri tempi, col gatto e la falena e la stoviglia semplice e fiorita e il commento dei cibi e Maddalena decrepita, e la siesta e la partita.

Per la partita, verso ventun'ore giungeva tutto l'inclito collegio politico locale: il molto Regio Notaio, il signor Sindaco, il Dottore; ma - poiché trasognato giocatore quei signori m'avevano in dispregio.

M'era più dolce starmene in cucina tra le stoviglie a vividi colori: tu tacevi, tacevo, Signorina: godevo quel silenzio e quegli odori tanto tanto per me consolatori, di basilico d'aglio di cedrina.

Maddalena con sordo brontolio disponeva gli arredi ben detersi, rigovernava lentamente ed io, già smarrito nei sogni più diversi, accordavo le sillabe dei versi sul ritmo eguale dell'acciottolio.

Sotto l'immensa cappa del camino (in me rivive l'anima d'un cuoco forse) godevo il sibilo del fuoco; la canzone d'un grillo canterino mi diceva parole, a poco a poco, e vedevo Pinocchio e il mio destino.

Vedevo questa vita che m'avanza: chiudevo gli occhi nei presagi grevi; aprivo gli occhi: tu mi sorridevi, ed ecco rifioriva la speranza!

Giungevano le risa, i motti brevi dei giocatori, da quell'altra stanza. (vv. 73-132)

In questi versi Gozzano tocca i suoi temi preferiti, tocca gli oggetti a lui più cari: 
- Descrizione di Felicita: vv. 73-90

- Il chiuso ambiente provinciale: vv. 91-96, 103-108

- L'odore del passato, la vita casalinga: vv. 109-114, 127-130

Oltre al distacco ironico della tradizione dell'amore romantico, è avvertibile la tematica del desiderio che si consuma e si sperde in immagini di evanescenza, o si scontra con una realtà che lo rende vano e impossibile, e quella di un erotismo nettamente connotato come infantile e adolescenziale. Un esempio chiaro sono i versi tratti da Le golose (vv.1-2): "Io sono innamorato di tutte le signore / che mangiano le paste nelle confetterie". In questa poesia (Gozzano 2008: 32-34) Gozzano gioca con il binomio desiderio - cibo, erotismo gestualità per dichiarare il suo amore per quelle giovani signore che ritornano bambine scegliendo le paste: "baciarvi ad una ad una / o belle bocche intatte / di giovani signore, / baciarvi nel sapore / di crema e cioccolatte" (vv. 46-50).

Tutto questo è Guido Gozzano, il cultore di "buone cose di pessimo gusto", l'amico di fanciulle e dame ottocentesche conosciute su vecchi album di sbiadite fotografie, il sentimentale giovane romantico che corteggia solo le donne che è sicuro di non sposare.

\subsection{Gozzano: il linguaggio}

$\mathrm{Ne} L$ 'amica di nonna Speranza il linguaggio aulico e quello prosastico si presentano entrambi fortemente caratterizzati. Nel primo caso si tratta della liricità romantica, nel secondo dello squallore quotidiano e naturalistico. Sono, entrambi, sottoposti a un processo di corrosione ironica. Sono inoltre continuamente accostati e mescolati, senza in realtà produrre forti scontri e tensioni, ma piuttosto combinazioni ironiche, ossimoriche: "l'iridi sincere / azzurre d'un azzurro di stoviglia" (La signorina Felicita, vv.83-84), "le buone cose di pessimo gusto" (L'amica di nonna Speranza, v. 2). Nei testi di Gozzano è frequente, specialmente ne La vita del rifugio e I Colloqui, l'uso dei segni indicanti durata, iterazione, rievocazione: avverbi come "ancora", "ormai", sintagmi come "non più", "altri tempi", "altre età" e soprattutto forme verbali contrassegnate dal prefisso "ri-", tutte portatrici di ripetizione, iterazione, ritorno: si pensi alla parola rinasco (L'amica di nonna Speranza, v. 14) che indica lo scarto temporale che permette l'ingresso nell'intemporalità della stampa ottocentesca.

Uno degli aspetti più caratteristici è l'uso della ripetizione, spesso ravvicinata, di una stessa parola o di un gruppo di parole, o sottolineata dalla posizione del verso, a ripresa di uno schema già fissato. In Gozzano l'uso dell'anafora diminuisce il valore semantico della parola, la alleggerisce e svuota di significato: "Ma bene... ma bene... ma bene" (L'amica di nonna Speranza, v. 57). Ci sono anche i casi di ripetizione di parole e frasi che vogliono rendere il linguaggio parlato: l'effetto in questo caso è quello di mostrare la vuota cerimoniosità sociale, la banalità, la mancanza di scambi veramente significativi. L'espediente della ripetizione di parole o di gruppi di parole, che in D'Annunzio era stato un mezzo a produrre l'estasi musicale (si pensi a $L a$ 
pioggia nel pineto (Budriesi 1990: 141-145) dove l'anafora piove rievoca il canto della natura che è il canto del poeta), nei crepuscolari sottolinea le ridondanze della lingua parlata: "Capenna... sicuro" (L'amica di nonna Speranza, vv.59-60).

Le rime spesso sono imperfette e, se non sono tali, se sottolineano veramente le parole rimanti, sono spesso in funzione ironica a creare abbinamenti dissacranti. Gozzano lascia alle sue spalle la tradizione pascoliana con la rima a catena (vedi la poesia Nebbia "lontane / frane, lontane / valeriane, lontane / pane, lontane / campane, lontane / cane" (Budriesi 1990: 72-73) o l'amore sensuale della parola di D'Annunzio, il recupero di termini arcaicizzanti e la scelta di vocaboli in funzione delle loro valenze evocativo - musicali. In Gozzano incontriamo rime che con toni talora sferzanti contribuiscono a dissolvere il mito superomistico dannunziano insieme a quello della pretesa funzione vaticinante della poesia: basti ricordare, per tutte, l'ardito e inaudito accostamento "camicie-Nietzsche", dissacrante e irrisorio nei confronti dei significati e soprattutto degli ideali convenuti. Uno schiaffo al passato, uno schiaffo all'intezione di D'Annunzio, suggestionato dalle idee di Nietzsche e Wagner, di creare l'opera artistica totale (Gesamtkunstwerk), in grado di educare le masse a un più alto senso del bello liberandole dal banale realismo del dramma borghese. Infallibile nella scelta delle parole, Gozzano è il primo che abbia giustapposto l'aulico con il profano:

Un esteta provinciale, a fondo parnasiano, un giovane piemontese malato, dannunziano, borghese, ma davvero piemontese e davvero borghese anche nel suo mondo [...] e fondó la sua poesia sullo choc che nasce tra una materia psicologicamente povera, frusta, apparentemente adatta ai soli toni minori, e una sostanza verbale ricca, gioiosa, estremamente compiaciuta di sé. (Montale 1997: 59)

Il linguaggio di Gozzano rompe gli schemi della tradizione precedente e introduce la parola realistica laddove il linguaggio poetico del primo e del secondo Ottocento si era mantenuto immune da forme popolari.

Le bellezze provinciali, i visi lentigginosi, le azzurre iridi domestiche, e signorine arcigne, visucci grami, donne quasi brutte e prive di lusinghe, dal volto quadro, cominciano a comparire soltanto nei poeti del primo Novecento, in Gozzano, in Moretti. E la realtà più familiare porta con sé (sia pure come controcanto o ironia verso il passato) parole di tutti i giorni. Decadono le parole sublimi. Gli scrittori scelgono di essere uomini comuni. (Beccaria 1988: 38)

\section{Conclusioni}

La stagione dei grandi letterati di fine Ottocento e inizi Novecento sembra essere ormai finita, la poesia incomincia ad essere relegata in un indefinito margine di importanza e i poeti si domandano quale sia il loro ruolo in questo 
nuovo contesto. Il confronto con i grandi del passato e con le epoche gloriose getta un senso di sconforto e impotenza, unitamente al rimpianto di non essere nati prima, in un tempo in cui la Poesia era tenuta ancora in grande considerazione. Cosa possono cantare $\mathrm{i}$ poeti che non sia già stato cantato? Quale grande ruolo e quale grande voce possono avere, se in realtà essi stessi per primi si sentono piccoli, minuti e in grado di emettere solo fievoli lamenti (o acute risate che nessuno vuole ascoltare)? Lasciamo la figura del superuomo dannunziana per cercare, come unica soluzione, il ripiegamento su se stessi, nel proprio piccolo mondo, facendo attenzione anche a quelle "piccole cose" che la grande poesia del passato ha sempre trascurato. Tutte le certezze di un tempo si sfaldano in modo inevitabile e il poeta inizia a guardare alla realtà con occhio quasi indifferente, malinconico e a volte ironico, teso a cogliere le sfumature quotidiane di una società in crisi. È il crepuscolo della grande Poesia che ripiega su più dimessi toni, ma contemporaneamente l'aurora di una nuova stagione letteraria.

Mentre la modernità inizia concretamente ad entrare nella vita dell'individuo, è necessario comprendere e metabolizzare nella propria intima essenza l'entità dei grandi cambiamenti innestati dallo sviluppo economico, tecnologico e industriale. I crepuscolari si approcciano alla realtà con uno sguardo quasi disorientato e avvertono il bisogno di rinchiudersi in un cantuccio riparato, pur continuando ad occhieggiare al mondo che prosegue la sua inarrestabile corsa verso il progresso. Essi si accorgono sventuratamente che il poeta ha ormai perduto la sua aureola e si adattano alla nuova condizione, ognuno seguendo la propria disposizione d'animo.

Con il suo nuovo modo di poetare, Gozzano ha dato inizio alla poesia del Novecento, vi regna il caos degli oggetti, il vocabolario di cose di poco conto. Si pensi al noto incipit de L'amica di nonna Speranza, il quale non solo costituisce una prova evidente dell'uso di un lessico quotidiano, ma testimonia anche, insieme a tutta la lirica, l'introduzione della narrazione e del parlato nella scrittura poetica. I versi gozzaniani vengono, dunque, contaminati dalla prosa attraverso il linguaggio colloquiale, il racconto, il dialogo.

Per concludere questo breve excursus, che vede nella figura di Gozzano e dei crepuscolari l'inizio della Poesia moderna, rilevanti sono le citazioni di Sanguineti e Mengaldo:

[...] il facile e ingannevole estetismo, in cui l'alto stile della tradizione perduta si risolve storicamente, si corromperà a contatto con la dura e provocante verità di un livello basso di quotidiana intonazione, anzi confesserà la propria intrinseca corruzione $\mathrm{e}$, intanto, per contro, l'insorgere realistico del prosaico, del parlato, denunzierà la miseria concreta delle cose, l'impossibilità di una redenzione estetica della vita. (Sanguineti 1966: 25)

Se insomma Gozzano fu maestro del controcanto prosaico e nella conseguente banalizzazione del linguaggio aulico, non lo fu meno 
nell'indicare la via di una sistematica rimotivazione tonale, e quasi specializzazione poetica, del lessico quotidiano, strategia che i poeti del Novecento hanno appreso soprattutto da lui. (Mengaldo 1978: 94)

"Saba comincia ad amare le parole trite, Montale a preferire gli erbosi fossi a bossi e ligustri e acanti dei poeti laureati" (Beccaria, 1988, p. 38); non bisogna sorprenderci dunque, considerato quanto detto, che autori della grandezza di Eugenio Montale e Cesare Pavese abbiano preso in considerazione l'esperimento crepuscolare per riportarlo nella loro poesia, facendo di Guido Gozzano la figura del poeta - ponte tra il decadentismo e poesia moderna italiana.

\section{Bibliografia citata}

BAUDELAIRE C. (1994). Les fleurs du mal. Paris: Garnier.

BECCARIA G. L. (1988). Italiano antico e nuovo. Milano: Garzanti.

Borgese G. A. (1910). Poesia crepuscolare, in La Spampa, giornale del $1^{\circ}$ settembre.

BUDRIESI A. (1990). Letteratura forma e modelli: il Novecento. Torino: Sei.

D’ANNUNZIO G. (1995). La figlia di Iorio. Milano: Garzanti.

FlaUberT G. (2009). Un coeur simple. Paris: Le livre de poche.

Gozzano G. (2008). Poesie e Prose. Milano: Feltrinelli.

Mengaldo P. V. (19789. Caratteristiche psicologiche e formali in Gozzano, da Poeti italiani del Novecento. Milano: Mondadori.

Montale E. (1997). Gozzano dopo trent'anni, Poesia. Milano: Mondadori.

PETRONIO G. (1937). I crepuscolari, Firenze: Sansoni.

PORCELli B. (1974). Gozzano originalità e plagi. Bologna: Patron Editore.

SANGUINETI E. (1966). Guido Gozzano. Indagini e letture. Torino: Einaudi.

Savoca G., Tropea M. (1985). Pascoli, Gozzano e i crepuscolari. Bari: Laterza.

\section{Bibliografia di riferimento}

\section{Per un ulteriore approfondimento sui crepuscolari:}

LIVI E. (1974). Dai simbolisti ai crepuscolari. Milano: IPL.

LUPERINI R. (1981). Il Novecento apparati ideologici ceto intellettuali e sistemi formali nella letteratura italiana contemporanea. Torino: Loescher.

SANGUINETI E.. (1976). Tra il liberty e il crepuscolarismo. Milano: Mursia.

Per un ulteriore approfondimento su Gozzano: vita, poesia e prosa, epistolario:

ASCIAMPRENER S. (1952). Lettere d'amore di Guido Gozzano e Amalia Guglielminetti. Milano: Garzanti.

CALCATERRA C. (1944). Con Guido Gozzano e altri poeti. Bologna: Zanichelli. 
Giorgia Marangon Guido Gozzano: dal Decadentismo al camino moderno della poesia italiana

Donato DE G. (1990). Lo spazio poetico di Guido Gozzano. Roma: Editori Riuniti.

LUGNANI L. (1973). Gozzano. Firenze:La nuova Italia.

RocCA A. (1980). Tutte le poesie. Milano: Mondadori. 